(C)2003 IEEE. Personal use of this material is permitted. However, permission to reprint/republish this material for advertising or promotional purposes or for creating new collective works for resale or redistribution to servers or lists, or to reuse any copyrighted component of this work in other works must be obtained from the IEEE. 


\title{
DETERMINISTIC CRAMÉR-RAO BOUND FOR SYMMETRIC PARAFAC MODEL WITH APPLICATION TO BLIND SPATIAL SIGNATURE ESTIMATION
}

\author{
Yue Rong ${ }^{1} \quad$ Sergiy A. Vorobyov ${ }^{1} \quad$ Alex B. Gershman ${ }^{1,2} \quad$ Nicholas D. Sidiropoulos $^{3}$ \\ ${ }^{1}$ Dept. of Communication Systems, University of Duisburg-Essen, Duisburg, Germany \\ ${ }^{2}$ Dept. of ECE, McMaster University, Hamilton, Ontario, Canada \\ ${ }^{3}$ Dept. of ECE, Technical University of Crete, Chania, Crete, Greece
}

\begin{abstract}
The symmetric PARAllel FACtor analysis (PARAFAC) model has found numerous applications in array signal processing and communications. In this paper, we derive the deterministic CramérRao Bound (CRB) for the symmetric RARAFAC model and illustrate the obtained results using an example with spatial signature estimation in sensor arrays.
\end{abstract}

\section{INTRODUCTION AND DATA MODEL}

A family of blind array processing algorithms including ESPRITlike method [1]-[2], Second Order Blind Identification (SOBI) algorithm [3]-[4] and blind spatial signature estimation method based on time-varying user power loading [5] exploit the models which essentially share the same structure called a symmetric PARAFAC model.

The CRB analysis for the methods based on the symmetric PARAFAC model is of great interest. In this paper, we derive such CRB in a closed form and illustrate the obtained results using an example with spatial signature estimation in sensor arrays.

Let an array of $K$ sensors receive the signals from $M$ narrowband sources. The $K \times 1$ snapshot vector of antenna array outputs can be written as

$$
\boldsymbol{y}(n)=\boldsymbol{A s}(n)+\boldsymbol{v}(n)
$$

where $\boldsymbol{A}=\left[\boldsymbol{a}_{1}, \ldots, \boldsymbol{a}_{M}\right]$ is the $K \times M$ complex matrix of the user spatial signatures, $\boldsymbol{a}_{m}=\left[a_{1, m}, \ldots, a_{K, m}\right]^{T}$ is the $K \times 1$ complex spatial signature of the $m$ th user, $s(n)=\left[s_{1}(n), \ldots\right.$, $\left.s_{M}(n)\right]^{T}$ is the $M \times 1$ complex vector of the user waveforms, $v(n)=\left[v_{1}(n), \ldots, v_{K}(n)\right]^{T}$ is the $K \times 1$ vector of additive spatially and temporally white complex Gaussian noise, and $(\cdot)^{T}$ denotes the transpose. Assuming that there is a block of $N$ snapshots available, the model (1) can be written as

$$
\boldsymbol{Y}=\boldsymbol{A S}+\boldsymbol{V}
$$

where $\boldsymbol{Y}=[\boldsymbol{y}(1), \ldots, \boldsymbol{y}(N)]$ is the $K \times N$ array data matrix, $\boldsymbol{S}=[\boldsymbol{s}(1), \ldots, \boldsymbol{s}(N)]$ is the $M \times N$ user waveform matrix, and $\boldsymbol{V}=[\boldsymbol{v}(1), \ldots, \boldsymbol{v}(N)]$ is the $K \times N$ sensor noise matrix.

Assuming that the user signals are uncorrelated with each other and sensor noise, the array covariance matrix of the received signals can be written as

$$
\boldsymbol{R}=\mathrm{E}\left\{\boldsymbol{y}(n) \boldsymbol{y}^{H}(n)\right\}=\boldsymbol{A} \boldsymbol{Q} \boldsymbol{A}^{H}+\sigma^{2} \boldsymbol{I}
$$

where $Q=\mathrm{E}\left\{s(n) s^{H}(n)\right\} \cdot$ is the diagonal covariance matrix of the signal waveforms, $\sigma^{2}$ is the sensor noise variance, $I$ is the identity matrix, and $(\cdot)^{H}$ denotes the Hermitian transpose.

\section{SYMMETRIC PARAFAC MODEL}

Often, it is required to estimate the matrix $\boldsymbol{A}$ in (2) based on the observations $\boldsymbol{Y}$ only. In the multiple user case, this is not possible to do with only one known covariance matrix (3) because the matrix $\boldsymbol{A}$ can be estimated from $\boldsymbol{R}$ only up to an arbitrary unknown unitary matrix. To provide a unique estimate of $\boldsymbol{A}$, several covariance matrices have to be used, see [1]-[5].

In this paper, following the approach of [5] with artificial user power loading, we assume that a set of covariance matrices is obtained by dividing uniformly the whole data block of $N$ snapshots into $P$ sub-blocks, each of $N_{\mathrm{s}}=\left\lfloor\frac{N}{P}\right\rfloor$ snapshots, where $\lfloor x\rfloor$ denotes the largest integer less than $x$. The transmitted power of each user is assumed to be fixed within each particular sub-block while is changed from one sub-block to another. Using such power loading scheme, we obtain that the received snapshots within any $p$ th sub-block correspond to the following covariance matrix

$$
\boldsymbol{R}(p)=\boldsymbol{A} \boldsymbol{Q}(p) \boldsymbol{A}^{H}+\sigma^{2} \boldsymbol{I}
$$

where $Q(p)$ is the diagonal covariance matrix of the user waveforms in the $p$ th sub-block and $p=1, \ldots, P$.

In practice, the noise power can be estimated and then subtracted from the covariance matrix (4). Let us stack the $P$ matrices $\boldsymbol{R}(p) \quad \sigma^{2} \boldsymbol{I}, p=1, \ldots, P$ together to form a three-way array $\underline{\boldsymbol{R}}$. This three-way array has a symmetry dictated by the symmetry of the matrices $\boldsymbol{R}(p) \quad \sigma^{2} \boldsymbol{I}$. The $(i, l, p)$ th element of such an array can be written as

$$
r_{i, l, p}=[\underline{\boldsymbol{R}}]_{i, l, p}=\sum_{m=1}^{M} a_{i, m} \nu_{m}(p) a_{l, m}^{*}
$$

where $\nu_{m}(p)=[Q(p)]_{m, m}$ is the power of the $m$ th user in the $p$ th sub-block and $(\cdot)^{*}$ denotes the complex conjugate. Defining the $P \times M$ matrix $P$ as

$$
\boldsymbol{P}=\left[\begin{array}{ccc}
\nu_{1}(1) & \ldots & \nu_{M}(1) \\
\vdots & \ddots & \vdots \\
\nu_{1}(P) & \ldots & \nu_{M}(P)
\end{array}\right]
$$


we have that $\boldsymbol{Q}(p)=\mathcal{D}_{p}\{\boldsymbol{P}\}$ for all $\dot{p}=1, \ldots, P$ where $\mathcal{D}_{p}\{\cdot\}$ is the operator that makes a diagonal matrix by selecting the $p$ th row and putting it on the main diagonal while putting zeros elsewhere.

\section{DETERMINISTIC CRAMÉR-RAO BOUND}

The model (1) for the $n$th sample of the $p$ th sub-block can be rewritten as

$$
\begin{aligned}
\boldsymbol{y}(p, n)=\boldsymbol{A} \boldsymbol{Q}^{1 / 2}(p) \tilde{s}(n)+ & \boldsymbol{v}(n), \\
n & =\left(\begin{array}{ll}
p & 1
\end{array}\right) N_{\mathrm{s}}+1, \ldots, p N_{\mathrm{s}}(7)
\end{aligned}
$$

where $\tilde{\boldsymbol{s}}(n)=\left[\bar{s}_{1}(n), \ldots, \tilde{s}_{M}(n)\right]^{T}=Q{ }^{1 / 2}(p) s(n)$ is the vector of normalized signal waveforms and the normalization is done so that all waveforms have unit powers.

Hence, the observations in the pth sub-block satisfy the following model

$$
\boldsymbol{y}(p, n) \sim \mathcal{C N}\left(\boldsymbol{\mu}(p, n), \sigma^{2} \boldsymbol{I}\right)
$$

where

$\boldsymbol{\mu}(p, n)=\boldsymbol{A} \boldsymbol{Q}^{1 / 2}(p) \tilde{\boldsymbol{s}}(n), \quad n=\left(\begin{array}{ll}p & 1\end{array}\right) N_{\mathrm{s}}+1, \ldots, p N_{\mathrm{s}}$

The unknown parameters of the model (7) are all the entries of $\boldsymbol{A}$, the diagonal elements of $\boldsymbol{Q}(p)(p=1, \ldots, P)$ and the noise power $\sigma^{2}$. Note, however, that the latter parameter is decoupled with the other parameters in the Fisher Information Matrix (FIM) [6]. Therefore, without loss of generality, $\sigma^{2}$ can be excluded from the vector of unknown parameters.

A delicate point regarding the CRB is the inherent permutation and scale ambiguity. To derive a meaningful $C R B$, we assume that the first row of $\boldsymbol{A}$ is normalized to $[1, \ldots, 1]_{1 \times M}$ (this removes the scaling ambiguity), and the first row of $\boldsymbol{P}$ is known and consists of distinct elements (which resolves the permutation ambiguity). Then, the $2\left(\begin{array}{ll}K & 1\end{array}\right) M \times 1$ real vector of the unknown parameters is given by

$$
\boldsymbol{\alpha}=\left[\boldsymbol{\alpha}_{2}^{T}, \ldots, \boldsymbol{\alpha}_{K}^{T}\right]^{T}
$$

where $\boldsymbol{\alpha}_{k}=\left[\operatorname{Re}\left\{\tilde{\boldsymbol{a}}_{k}\right\}^{T}, \operatorname{Im}\left\{\tilde{\boldsymbol{a}}_{k}\right\}^{T}\right]^{T}$ and $\tilde{\boldsymbol{a}}_{k}=\left[a_{k, 1}, \ldots, a_{k, M}\right]^{T}$ The $\left(\begin{array}{ll}P & 1\end{array}\right) M \times 1$ vector of nuisance parameters can be expressed as

$$
\boldsymbol{\zeta}=[\overline{\boldsymbol{p}}(2), \ldots, \tilde{\boldsymbol{p}}(P)]^{T}
$$

where $\tilde{p}(p)$ is the $p$ th row of the matrix $P$

$$
\text { Using (10) and (11), the }\left(2\left(\begin{array}{ll}
K & 1
\end{array}\right) M+\left(\begin{array}{ll}
P & 1
\end{array}\right) M\right) \times 1 \text { real }
$$
vector of unknown parameters can be written as

$$
\boldsymbol{\theta}=\left[\boldsymbol{\alpha}^{T}, \boldsymbol{\zeta}^{T}\right]^{T}
$$

THEOREM: The $\left(2\left(\begin{array}{ll}K & 1\end{array}\right) M+\left(\begin{array}{ll}P & 1\end{array}\right) M\right) \times\left(2\left(\begin{array}{ll}K & 1\end{array}\right) M+\right.$ $\left(\begin{array}{ll}P & 1\end{array}\right) M$ ) FIM is given by

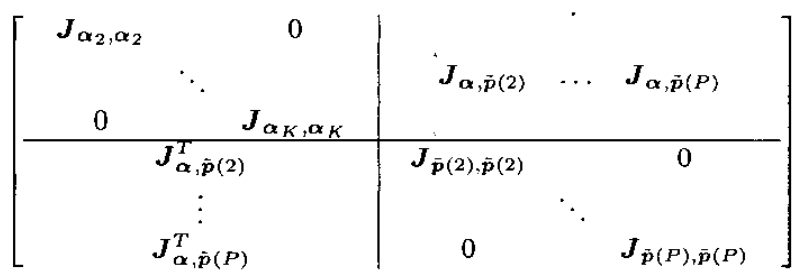

where

$$
\begin{aligned}
& J_{\boldsymbol{\alpha}_{2}, \boldsymbol{\alpha}_{2}}=\cdots=J_{\boldsymbol{\alpha}_{K}, \boldsymbol{\alpha}_{K}} \\
& =\frac{2}{\sigma^{2}}\left[\begin{array}{lc}
\operatorname{Re}\left\{\mathbf{\Upsilon}^{H} \mathbf{Y}\right\} & \operatorname{Im}\left\{\mathbf{\Upsilon}^{H} \mathbf{Y}\right\} \\
\operatorname{Im}\left\{\mathbf{\Upsilon}^{H} \mathbf{Y}\right\} & \operatorname{Re}\left\{\mathbf{\Upsilon}^{H} \mathbf{\Upsilon}\right\}
\end{array}\right] \\
& J_{\tilde{\boldsymbol{p}}(p), \tilde{p}(p)}=\frac{2}{\sigma^{2}} \operatorname{Re}\left\{(G(p))^{H} \boldsymbol{G}(p)\right\} \\
& \boldsymbol{J}_{\boldsymbol{\alpha}, \overline{\boldsymbol{p}}(p)}=\frac{2}{\sigma^{2}}\left(\boldsymbol{I}_{K} \quad 1 \otimes \tilde{\boldsymbol{F}}(p)\right) \tilde{\boldsymbol{H}}(p) \\
& \boldsymbol{\Upsilon}=\left[\begin{array}{ccc}
\boldsymbol{f}_{1}(1) & \ldots & \boldsymbol{f}_{M}(1) \\
\vdots & \ddots & \vdots \\
\boldsymbol{f}_{1}(P) & \ldots & \boldsymbol{f}_{M}(P)
\end{array}\right] \\
& \boldsymbol{G}(p)=\left[\begin{array}{ccc}
\boldsymbol{h}_{1,1}(p) & \ldots & \boldsymbol{h}_{1, M}(p) \\
\vdots & \ddots & \vdots \\
\boldsymbol{h}_{K, 1}(p) & \ldots & \boldsymbol{h}_{K, M}(p)
\end{array}\right] \\
& \tilde{\boldsymbol{F}}(p)=\left[\begin{array}{cc}
\operatorname{Re}\left\{\boldsymbol{F}^{H}(p)\right\} & \operatorname{Im}\left\{\boldsymbol{F}^{H}(p)\right\} \\
\operatorname{Im}\left\{\boldsymbol{F}^{H}(p)\right\} & \operatorname{Re}\left\{\boldsymbol{F}^{H}(p)\right\}
\end{array}\right] \\
& \boldsymbol{F}(p)=\left[\boldsymbol{f}_{1}(p), \ldots, \boldsymbol{f}_{M}(p)\right] \\
& \overline{\boldsymbol{H}}(p)=\left[\tilde{\boldsymbol{H}}_{2}^{T}(p), \ldots, \tilde{\boldsymbol{H}}_{K}^{T}(p)\right]^{T} \\
& \tilde{\boldsymbol{H}}_{k}(p)=\left[\begin{array}{l}
\operatorname{Re}\left\{\boldsymbol{H}_{k}(p)\right\} \\
\operatorname{Im}\left\{\boldsymbol{H}_{k}(p)\right\}
\end{array}\right] \\
& \boldsymbol{H}_{k}(p)=\left[\boldsymbol{h}_{k, 1}(p), \ldots, \boldsymbol{h}_{k, M}(p)\right] \\
& f_{m}(p)=\left[\sqrt{\nu_{m}(p)} \bar{s}_{m}\left(\begin{array}{ll}
(p & 1) N_{\mathrm{s}}+1
\end{array}\right),\right. \\
& \left.\ldots, \sqrt{\nu_{m}(p)} \tilde{s}_{m}\left(p N_{\mathrm{s}}\right)\right]^{T} \\
& h_{k, m}(p)=\left[\frac{a_{k, m} \tilde{s}_{m}\left((p \quad 1) N_{\mathrm{s}}+1\right)}{2 \sqrt{\nu_{m}(p)}},\right. \\
& \left.\ldots, \frac{a_{k, m} \tilde{s}_{m}\left(p N_{s}\right)}{2 \sqrt{\nu_{m}(p)}}\right]^{T}
\end{aligned}
$$

and $\otimes$ denotes the Kronecker matrix product.

The $\left(\begin{array}{ll}K & 1\end{array}\right) M \times\left(\begin{array}{ll}K & 1\end{array}\right) M$ spatial signature-related block of the CRB matrix is given in the closed form as

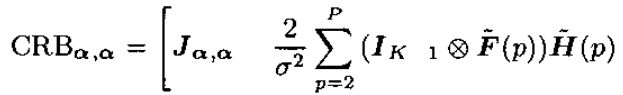

$$
\begin{aligned}
& \left.\times\left[\operatorname{Re}\left\{G^{H}(p) \boldsymbol{G}(p)\right\}\right]^{1} \tilde{\boldsymbol{H}}^{H}(p)\left(\boldsymbol{I}_{K} \quad 1 \otimes \tilde{\boldsymbol{F}}(p)\right)^{H}\right]^{1}
\end{aligned}
$$

where the upper-left block of the FIM can be expressed as

$$
J_{\boldsymbol{\alpha}, \boldsymbol{\alpha}}=\frac{2}{\sigma^{2}} I_{K} \quad{ }_{1} \otimes\left[\begin{array}{cc}
\operatorname{Re}\left\{\mathbf{\Upsilon}^{H} \mathbf{\Upsilon}\right\} & \operatorname{Im}\left\{\boldsymbol{\Upsilon}^{H} \mathbf{\Upsilon}\right\} \\
\operatorname{Im}\left\{\mathbf{\Upsilon}^{H} \boldsymbol{\Upsilon}\right\} & \operatorname{Re}\left\{\boldsymbol{\Upsilon}^{H} \boldsymbol{\Upsilon}\right\}
\end{array}\right]
$$

Proof: The $(l, k)$ th element of the FIM is given by [6]

$$
\begin{aligned}
& \mathrm{FIM}_{l, k}=\frac{2}{\sigma^{2}} \\
\times & \sum_{p=1}^{P} \sum_{n=(p}^{p N_{s}} \operatorname{Re}\left(\frac{\partial \boldsymbol{\mu}^{H}(p, n)}{\partial \theta_{l}} \frac{\partial \boldsymbol{\mu}(p, n)}{\partial \theta_{k}}\right)
\end{aligned}
$$


Using (9) along with (27), we have

$$
\begin{aligned}
\frac{\partial \mu(p, n)}{\partial \operatorname{Re}\left\{a_{k, m}\right\}} & =\sqrt{\nu_{m}(p)} \bar{s}_{m}(n) e_{k} \\
\frac{\partial \mu(p, n)}{\partial \operatorname{Im}\left\{a_{k, m}\right\}} & =j \sqrt{\nu_{m}(p)} \bar{s}_{m}(n) e_{k} \\
\frac{\partial \mu(p, n)}{\partial \nu_{m}(p)} & =\left[\frac{a_{1, m} \bar{s}_{m}(n)}{2 \sqrt{\nu_{m}(p)}}, \ldots, \frac{a_{K, m} \tilde{s}_{m}(n)}{2 \sqrt{\nu_{m}(p)}}\right]^{T}
\end{aligned}
$$

where $e_{k}$ is the vector containing one in the $k$ th position and zeros elsewhere.

Using (28) and (29) along with (27) we obtain that

$$
\begin{aligned}
& J_{\operatorname{Re}\left\{a_{k, m}\right\}, \operatorname{Re}\left\{a_{k, l}\right\}}=J_{\operatorname{Im}\left\{a_{k, m}\right\}, \operatorname{Im}\left\{a_{k, l}\right\}} \\
= & \frac{2}{\sigma^{2}} \sum_{p=1}^{P} \sum_{n=(p}^{p N_{\mathrm{s}}} \operatorname{Re}\left\{\sqrt{\nu_{m}(p) \nu_{l}(p)} \tilde{s}_{m}^{*}(n) \tilde{s}_{l}(n)\right\} \\
= & \frac{2}{\sigma^{2}} \operatorname{Re}\left\{\xi_{m}^{H} \xi_{l}\right\}
\end{aligned}
$$

where $\boldsymbol{\xi}_{m}=\left[\boldsymbol{f}_{m}^{T}(1), \ldots, \boldsymbol{f}_{m}^{T}(P)\right]^{T}$

Similarly,

$$
\begin{aligned}
J_{\operatorname{Im}\left\{a_{k, m}\right\}, \operatorname{Re}\left\{a_{k, l}\right\}} & =J_{\operatorname{Re}\left\{a_{k, m}\right\}, \operatorname{Im}\left\{a_{k, l}\right\}} \\
& =\frac{2}{\sigma^{2}} \operatorname{Im}\left\{\boldsymbol{\xi}_{m}^{H} \xi_{l}\right\}
\end{aligned}
$$

Therefore,

$$
\begin{aligned}
& \boldsymbol{J}_{\operatorname{Re}\left\{\boldsymbol{\alpha}_{k}\right\}, \operatorname{Re}\left\{\boldsymbol{\alpha}_{k}\right\}}=\boldsymbol{J}_{\operatorname{Im}\left\{\boldsymbol{\alpha}_{k}\right\}, \operatorname{Im}\left\{\boldsymbol{\alpha}_{k}\right\}} \\
= & \frac{2}{\sigma^{2}}\left[\begin{array}{ccc}
\operatorname{Re}\left\{\boldsymbol{\xi}_{1}^{H} \boldsymbol{\xi}_{1}\right\} & \ldots & \operatorname{Re}\left\{\boldsymbol{\xi}_{1}^{H} \boldsymbol{\xi}_{M}\right\} \\
\vdots & \ddots & \vdots \\
\operatorname{Re}\left\{\boldsymbol{\xi}_{M}^{H} \boldsymbol{\xi}_{1}\right\} & \ldots & \operatorname{Re}\left\{\boldsymbol{\xi}_{M}^{H} \boldsymbol{\xi}_{M}\right\}
\end{array}\right] \\
= & \frac{2}{\sigma^{2}} \operatorname{Re}\left\{\boldsymbol{\Upsilon}^{H} \boldsymbol{\Upsilon}\right\}
\end{aligned}
$$

and

$$
\begin{aligned}
& \boldsymbol{J}_{\operatorname{Im}\left\{\boldsymbol{\alpha}_{k}\right\}, \operatorname{Re}\left\{\boldsymbol{\alpha}_{k}\right\}}= \\
= & \frac{2}{\sigma^{2}}\left[\begin{array}{ccc}
\operatorname{Im}\left\{\boldsymbol{\xi}_{1}^{H} \boldsymbol{\xi}_{1}\right\} & \ldots & \operatorname{Im}\left\{\boldsymbol{\xi}_{1}^{H} \boldsymbol{\xi}_{M}\right\} \\
\vdots & \ddots & \vdots \\
\operatorname{Im}\left\{\boldsymbol{\xi}_{M}^{H} \boldsymbol{\xi}_{1}\right\} & \ldots & \operatorname{Im}\left\{\boldsymbol{\xi}_{M}^{H} \boldsymbol{\xi}_{M}\right\}
\end{array}\right] \\
= & \frac{2}{\sigma^{2}} \operatorname{Im}\left\{\mathbf{\Upsilon}^{H} \mathbf{\Upsilon}\right\}
\end{aligned}
$$

Using (33) and (34), we obtain (13). Note that the right-hand side of (13) does not depend on the index $k$. Hence,

$$
\begin{aligned}
\boldsymbol{J}_{\boldsymbol{\alpha}, \boldsymbol{\alpha}} & =\left[\begin{array}{ccc}
J_{\boldsymbol{\alpha}_{2}, \boldsymbol{\alpha}_{2}} & & 0 \\
& \ddots & \\
0 & J_{\alpha_{K}, \boldsymbol{\alpha}_{K}}
\end{array}\right] \\
& =\frac{2}{\sigma^{2}} \boldsymbol{I}_{K} \quad 1 \otimes\left[\begin{array}{ll}
\operatorname{Re}\left\{\mathbf{\Upsilon}^{H} \mathbf{\Upsilon}\right\} & \operatorname{Im}\left\{\mathbf{\Upsilon}^{H} \mathbf{\Upsilon}\right\} \\
\operatorname{Im}\left\{\mathbf{\Upsilon}^{H} \mathbf{\Upsilon}\right\} & \operatorname{Re}\left\{\mathbf{\Upsilon}^{H} \mathbf{\Upsilon}\right\}
\end{array}\right]
\end{aligned}
$$

Next, using (30) along with (27) we can write for $p=2, \ldots, P$ and $m, l=1, \ldots, M$

$$
\begin{aligned}
& {\left[J_{\tilde{p}(p), \tilde{p}(p)]_{m, l}=\frac{2}{\sigma^{2}}}\right.} \\
\times & \sum_{n=(p \quad i) N_{s}+1}^{p N_{s}} \sum_{k=1}^{K} \operatorname{Re}\left\{\frac{\left(a_{k, m} \tilde{s}_{m}(n)\right)^{*}}{2 \sqrt{\nu_{m}(p)}} \frac{a_{k, l} \tilde{s}_{l}(n)}{2 \sqrt{\nu_{l}(p)}}\right\} \\
= & \frac{2}{\sigma^{2}} \operatorname{Re}\left\{c_{m}^{H}(p) c_{l}(p)\right\}
\end{aligned}
$$

where $c_{m}(p)=\left[\boldsymbol{h}_{1, m}^{T}(p), \ldots, \boldsymbol{h}_{K, m}^{T}(p)\right]^{T}$. Stacking all $M^{2}$ elements given by $(36)$ in one matrix we have for $p=2, \ldots, P$

$$
\begin{aligned}
& J_{\hat{p}(p), \tilde{p}(p)}=\frac{2}{\sigma^{2}} \\
\times & {\left[\begin{array}{ccc}
\operatorname{Re}\left\{c_{1}^{H}(p) c_{1}(p)\right\} & \ldots & \operatorname{Re}\left\{c_{1}^{H}(p) c_{M}(p)\right\} \\
\vdots & \ddots & \vdots \\
\operatorname{Re}\left\{c_{M}^{H}(p) c_{1}(p)\right\} & \ldots & \operatorname{Re}\left\{c_{M}^{H}(p) c_{M}(p)\right\}
\end{array}\right] } \\
= & \frac{2}{\sigma^{2}} \operatorname{Re}\left\{G^{H}(p) G(p)\right\}
\end{aligned}
$$

Finally, using (28), (29), and (30) along with (27) we can write for $p=2, \ldots P ; k=2, \ldots, K$, and $m, l=1, \ldots, M$

$$
\begin{aligned}
& {\left[\boldsymbol{J}_{\left.\operatorname{Re}\left\{\boldsymbol{a}_{k}\right\}, \tilde{\boldsymbol{p}}(p)\right]_{m, l}}=\frac{2}{\sigma^{2}}\right.} \\
\times & \sum_{n=(p}^{p N_{\mathrm{s}}} \operatorname{Re}\left\{\frac{1}{2} \frac{\sqrt{\nu_{m}(p)}}{\sqrt{\nu_{l}(p)}} \tilde{s}_{m}^{*}(n) a_{k, l} \tilde{s}_{l}(n)\right\} \\
= & \frac{2}{\sigma^{2}} \operatorname{Re}\left\{\boldsymbol{f}_{m}^{H}(p) \boldsymbol{h}_{k, l}(p)\right\} \\
\times & \sum_{\left.\mathrm{Im}\left\{\boldsymbol{a}_{k}\right\}, \tilde{p}(p)\right]_{m, l}=\frac{2}{\sigma^{2}}} \operatorname{Re}\left\{j \frac{1}{2} \frac{\sqrt{\nu_{m}(p)}}{\sqrt{\nu_{l}(p)}} \tilde{s}_{m}^{*}(n) a_{k, l} \tilde{s}_{l}(n)\right\} \\
= & \frac{2}{\sigma^{2}} \operatorname{Im}\left\{\boldsymbol{f}_{m}^{H}(p) h_{k, l}(p)\right\}
\end{aligned}
$$

Collecting all $\left(\begin{array}{ll}K & 1\end{array}\right) M^{2}$ elements given by (38) and $\left(\begin{array}{ll}K & 1\end{array}\right) M^{2}$ elements given by (39) in one matrix, we obtain for $p=2, \ldots, P$

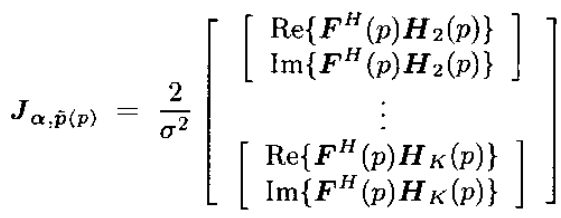

Observing that

$$
\left[\begin{array}{c}
\operatorname{Re}\left\{\boldsymbol{F}^{H}(p) \boldsymbol{H}_{k}(p)\right\} \\
\operatorname{Im}\left\{\boldsymbol{F}^{H}(p) \boldsymbol{H}_{k}(p)\right\}
\end{array}\right]=\tilde{\boldsymbol{F}}(p) \tilde{\boldsymbol{H}}_{k}(p)
$$

we can further simplify (40) to

$$
\boldsymbol{J}_{\boldsymbol{\alpha}, \tilde{\boldsymbol{p}}(p)}=\frac{2}{\sigma^{2}}\left(\boldsymbol{I}_{K} \quad{ }_{1} \otimes \tilde{\boldsymbol{F}}(p)\right) \tilde{\boldsymbol{H}}(p)
$$

Also, note that

$$
\boldsymbol{J}_{\boldsymbol{\alpha}, \tilde{p}(p)}^{T}=\boldsymbol{J}_{\tilde{p}(p), \boldsymbol{\alpha}}
$$




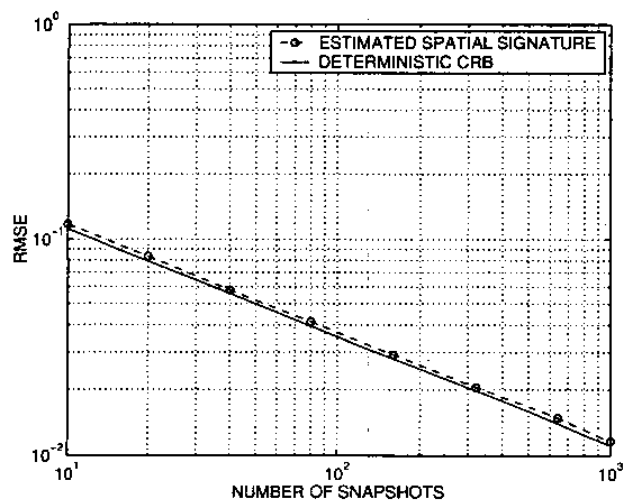

Fig. 1. CRB and RMSE versus $N$. (24).

Using (35), (37), (42) and (43) we obtain the expressions (13)-

Computing the CRB for $\theta$ requires the inverse of the $(2(K$ 1) $\left.M+\left(\begin{array}{ll}P & 1\end{array}\right) M\right) \times\left(2\left(\begin{array}{ll}K & 1\end{array}\right) M+\left(\begin{array}{ll}P & 1\end{array}\right) M\right)$ FIM matrix. Our objective is to obtain the CRB associated with the vector parameter $\alpha$ only, avoiding the inverse of the full FIM matrix. Exploiting the fact that the lower-right sub-block of the FIM is a block-diagonal matrix and using the partitioned matrix inversion lemma (see [6], p. 572), after some algebra we obtain (25)-(26) and the proof is complete.

\section{SIMULATIONS}

In order to test the derived CRB we consider a simple example with spatial signature estimation of a single user and assume that the BPSK signal impinges on the linear array of 4 sensors and unknown geometry from $\theta=50^{\circ}$ relative to the broadside direction. It is well known that in the single-user case, a single covariance matrix is sufficient to guarantee the uniqueness of the spatial signature estimate which is given by the principal eigenvector of the sample covariance matrix $\hat{R}$.

We compare the Root-Mean-Square Error (RMSE) performance of such a principal eigenvector-based estimator with the derived CRB. The RMSE is computed as

$$
\operatorname{RMSE}=\sqrt{\frac{1}{\bar{L} \bar{K} \sum_{l=1}^{L}\|\hat{a}(l) \quad a\|_{F}^{2}}}
$$

where $L=100$ is the number of independent simulation runs and $\hat{a}(l)$ is the estimate of $a$ obtained in the $l$ th run. Note that the scaling ambiguity is eliminated by normalizing $\hat{a}(l)$ with respect to the first (reference) sensor. The CRB is computed as

$$
\mathrm{CRB}=\sqrt{\frac{1}{K 1} \operatorname{Tr}\left\{\mathrm{CRB}_{\alpha, \alpha}\right\}}
$$

Figure 1 displays the RMSE and the CRB versus the number of snapshots $N$ for the Signal-to-Noise Ratio (SNR) equal to $10 \mathrm{~dB}$. Figure 2 shows the same quantities versus the SNR for $N=100$.

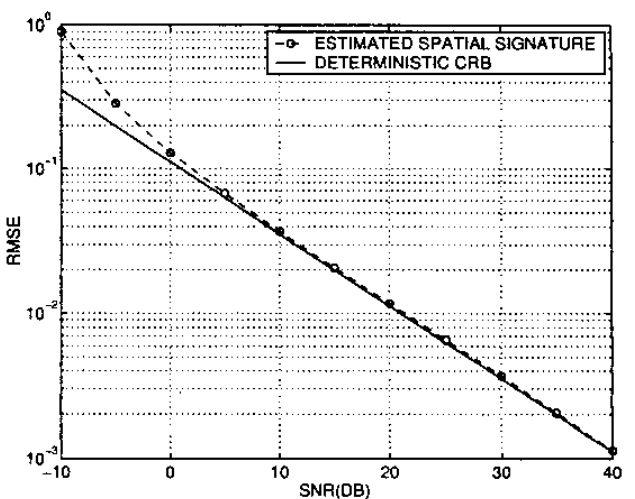

Fig. 2. CRB and RMSE versus SNR.

It can be seen that the principal eigenvector-based spatial signature estimator approaches CRB at high SNR. This validates our CRB analysis.

\section{CONCLUSIONS}

The closed-form expressions for the deterministic CRB for the symmetric PARAFAC model have been derived. The simulation example with blind spatial signature estimation illustrates and validates our CRB analysis.

\section{REFERENCES}

[1] D. Astèly, A. L. Swindlehurst and B. Ottersten, "Spatial signature estimation for uniform linear arrays with unknown receiver gains and phases," IEEE Trans. Signal Processing, vol. 47, pp. 2128-2138, Aug. 1999.

[2] M. K. Tsatsanis and C. Kweon, "Blind source separation of non-stationary sources using second-order statistics," in Proc. 32nd Asilomar Conf. Signals, Systems and Computers, Pacific Grove, CA, Nov. 1998, vol. 2, pp. 1574 -1578.

[3] A. Belouchrani, K. Abed-Meraim, J-F. Cardoso and E. Moulines, "A blind source separation technique using second-order statistics," IEEE Trans. on Signal Processing, vol. 45, No.2, pp. 434-444, Feb. 1997.

[4] A. Yeredor, "Non-orthogonal joint diagonalization in the least-squares sense with application in blind source separation," IEEE Trans. Signal Processing, vol. 50, pp. 15451553 , July 2002.

[5] Y. Rong, S. A. Vorobyov, A. B. Gershman and N. D. Sidiropoulos, "Blind spatial signature estimation using time-varying user power loading and parallel factor analysis," Proc. IEEE VTC'03 Fall, Orlando, USA, Oct. 2003 (also submitted to IEEE Trans. Signal Processing).

[6] S. M. Kay, Fundamentals of Statistical Signal Processing: Estimation Theory, Englewood Cliffs, NJ: Prentice-Hall, 1993. 